\title{
Assessment of Behavior and Attitude of Academic Community During Covid-19 Contingency
}

\author{
Carrasco Omar $\mathrm{F}^{1,2}$, Fajardo Dolci German ${ }^{1}$, Olaiz Fernández Gustavo ${ }^{1}$, Juárez Arturo ${ }^{1,2}$, Vicuña \\ Félix $^{1,2}$, Muñoz Arturo', Veloz Corona Quetzallii ${ }^{2,3}$ and Juárez Olguin Hugo*2,3 \\ ${ }^{1}$ Centro de Investigación en Política, Población y Salud, Facultad de Medicina UNAM, Mexico
}

${ }^{2}$ Departamento de Farmacología, Facultad de Medicina UNAM, Mexico

${ }^{3}$ Laboratorio de Farmacología, Instituto Nacional de Pediatría, Mexico

*Corresponding author: Hugo Juárez Olguín, Laboratorio de Farmacología, Instituto Nacional de Pediatría, Mexico.

To Cite This Article: Hugo Juárez Olguín, Assessment of Behavior and Attitude of Academic Community During Covid-19 Contingency. 2020 - 10(2). AJBSR.MS.ID.001486. DOI: 10.34297/AJBSR.2020.10.001486.

Received: 眥 August 31, 2020; Published: 制 September 03, 2020

\begin{abstract}
The survey about the behavior and attitude of an academy community was carried out to know their preventive measures and response caused by SARS-CoV-2 infection. In the period between March 26 and May 31, 2020, data from "Survey on the Follow-up of the Community of the Medical Faculty" were collected using Lime Survey version 4.2.5 in the CIPPS Survey Service. The use of mouth and face mask in the respondents barely exceeded $70 \% .10 \%$ of the respondents have chronic diseases such as hypertension and obesity. $95 \%$ reported constant hygienic measure of hand-wash with soap and water, while more than $94 \%$ have respected physical-space distance. $20 \%$ of the survey population showed respiratory symptoms, which fell to $5 \%$ in the last 9 weeks. The most frequently reported symptoms were headache, cough, odynophagia, rhinorrhea, asthenia, anosmia and symptoms associated with respiratory tract. Less than $1 \%$ required medical attention, using non-steroid anti-inflammatory drugs. The analysis of the academic community survey depicts the importance of community discipline and the interest in collective health. The dissemination of useful information to prevent infections such as the use of mouth and face masks, physical-space distance and direct medical orientation guidance should continue to be strengthened.
\end{abstract}

Keywords: Attitudes; University Community; COVID-19; Survey

\section{Introduction}

Airway infections are the cause of more than 4 million deaths a year and approximately $40 \%$ of them are produced by viral infections Troeger [1]. Acute respiratory diseases produced by viruses have a great impact and importance for global public health. Severe acute respiratory syndrome (SARS) is a respiratory disease provoked by a coronavirus, named SARS-CoV-associated coronavirus. In 2003, the World Health Organization (WHO) [2] reported an accumulated 8,439 infected people by SARS-CoV with a toll of 812 deaths. The outbreak, which started in China spread to 30 countries and was successfully suppressed, thanks to timely detection measures and isolation of cases WHO [3]. From this moment on, the possible future transmissions of the virus were announced. Likewise, a call was made to collaborate in the preparation of research protocols and case management, emphasizing the restructuring and strengthening of the health systems to cope in disease care. In December 2019, in Wuhan province of Hubei, China, the first case of pneumonia of unknown etiology was reported (JAMA 2020). On January 7 , the pathogen was identified as a new coronavirus and by January 30, the WHO declared the Severe Acute Respiratory Syndrome 2 (SARS-Cov-2) a health emergency of global concern. Since then, the world has been fighting tooth and nail to come up with specific drug to attack the virus to no avail Echeverria et al. [4]. SARS-CoV-2 is a virus with a high contagion rate that has collapsed several health systems in the world. The alarming speed of the contagion, aided by facilities in global travel, took the virus to virtually all the continents of the world, in such a way that by June 
26,2020 , a staggering number of confirmed cases have risen to $9,683,414$ infected people worldwide, with a toll of 491,095 deaths. On the same date, in Mexico, 202,951 confirmed cases with a total of 25,060 deaths were reported J Hopkins [5].

Based on the virulence and the speed of spread, a vehement call was made to the health ministries of all the affected countries around the world to reinforce measures for the control of SARSCoV-2, which, with no existing specific treatment, should be based on adoption of effective preventive measures to reduce the contagions and propagation of the virus. In the light of SARS-CoV-2 transmission mechanisms based on close contact and aerosols (Interim Guidance 2020), the actions of prevention are focused on disinfection and hygiene. In a study performed in 2018 with the objective of determining the survival time of SARS and MERS (Meddle East Respiratory Syndrome), it was reported that SARS-CoV can survive up to 72 hours on stainless steel and plastic and 24 hours on surfaces such as copper. Some of the measures recommended by the WHO are: maintenance of person-to-person physical-space distance of at least 1 meter; frequent hand-wash with alcohol when the hand is visibly not dirty and with water and soap on the contrary; avoidance of eyes, nose and mouth touching; correct hygienic coughing and sneezing which entail covering the face with the internal face of the elbow or with disposable hygienic paper which should be disposed immediately after use; wearing mouth and face masks in case of respiratory symptoms and hand wash on removing or getting rid of it; routine cleaning and disinfection of all surroundings and other frequently touched surfaces. In addition to these measures aimed at the entire population, the protection of the health professionals in charge of the COVID-19 patients is required. In this case, airborne disinfections based on application of environmental aerosols and support treatment should be strictly undertaken Standard Precautions 2020. Not only have the health authorities been urged to implement the aforementioned measures, but also to cover the needed resources which the protocol to mitigate the spread of a pandemics demands, by actively searching for contacts with confirmatory tests. The purpose of containment measures is to suspend or impede the development of crowding activities and encourage social-distancing, closure of schools as well as non-essential businesses and commerce Mitigation [6]. In this health emergency, it is difficult to foresee which are the most effective measures and how to implement them in order to reduce the number of infected cases. For this, estimations with mathematical models has been formulated and applied in the observation of the behavior of the contagion or spread of the infection with social-distancing measures. A study performed in Singapore, one of the countries with less number of deaths and confirmed cases associated with SARS-CoV-2, showed a simulation of 100 infected cases in 80 days in different scenarios J Hopkins [5].
In the first scenario, there was no intervention and data on infected cases registered a total of 279,000 people which corresponds to $7.4 \%$ of the population. In the second scenario with the population in quarantine, the result of confirmed case was 15,000 . In the third simulation with closure of schools and remote working, the number of contagion was 10,000 cases on day 80. In the fourth scenario, involving the combination of the aforementioned measures, the confirmed cases were 1,800 on day 80 Koo et al. [7]. Another study carried out with EnerPol platform in Swiss population including interventions such as closure of schools, activities, limitation of public transport and social distancing from February 22 to April 11, reported that $42 \%$ of the population were infected without government intervention compared to $1 \%$ of the population infected with timely intervention Reza et al. [8]. The objective of the present work was to evaluate the behavior and attitude of the students and staffs of the Faculty of Medicine (FacMed), Universidad Nacional Autónoma de México (UNAM) in the implementation of preventive measures, as well as adhesion and response to them, in effort to decrease the fast spread of the disease caused by SARS-Cov- 2 .

\section{Methods}

In the period between March 26 and May 31, 2020, data from "Survey on the Follow-up of the Community of FacMed" were collected using Limesurvey version 4.2.5 in the CIPPS Survey Service. All the personnel of Faculty of Medicine at Universidad Nacional Autónoma de Mexico (UNAM), participated in the survey, including students, professors and academics. The information collected were: general data, age, gender, comorbidities, history of travel in the last 14 days, confinement-breaking activities, measures taken during confinement-breaking activities and on returning, appearance of respiratory symptoms, medications used and sought for medical attention. The analysis included all the members of the Community of FacMed and the people living with them. The invitations were sent on March 26, 2020 by social media, emails and faculty website. The data were stored in SQL Server Enterprise 2017 database and were downloaded for their statistical analysis using ODBC STATA 16 IC.

\section{Results}

In Table 1, the number of people who responded the survey in the period between March 26, 2020 and May 31, 2020 are shown. In the Table 2, the demographic characteristics of the respondents can be observed. Figure 2 shows the age distribution of the members of FacMed community and the people who live in it. In the first week of confinement, $19.9 \%$ of the respondents had to leave their house and during the rest of the confinement, this exit from home came down. (Table 3) shows the percentage of the community that left home during the confinement. For the first 
three recommendations of the WHO such as constant hand-wash with water and soap, quarantine keeping and the use of mouth mask, the answered of the respondents were complete obeisance to the recommendation. (Table 4) describes the rest of the sanitary measures that the community implemented to avoid the spread of COVID-19. Of the people who had to leave their houses, the answers given were mainly for work and for shopping for food and the transport means used were mainly buses. The rest of the reasons and the transport means used are shown in Table 5. Most

Table 1: Number of participants in in the survey per week.

\begin{tabular}{|c|c|c|}
\hline \multicolumn{2}{|c|}{ Week } & Participants \\
\hline 26-Mar & 3-Apr & 14699 \\
\hline $4-\mathrm{Apr}$ & $10-\mathrm{Apr}$ & 13485 \\
\hline $11-\mathrm{Apr}$ & $17-\mathrm{Apr}$ & 11577 \\
\hline $18-\mathrm{Apr}$ & $24-\mathrm{Apr}$ & 8374 \\
\hline $25-\mathrm{Apr}$ & 1-May & 8114 \\
\hline 2-May & 8-May & 6334 \\
\hline 9-May & 15-May & 8490 \\
\hline $16-$ May & 22-May & 8732 \\
\hline 23-May & 31-May & 91409 \\
\hline
\end{tabular}

Table 2: Demographic characteristics of the population that participated in the survey.

\begin{tabular}{|c|c|c|c|}
\hline Characteristics & Men & Women & Total \\
\hline Number & 9345 & 11653 & \multirow{2}{*}{20998} \\
\hline (Percentage) & $-44.50 \%$ & $-55.50 \%$ & 36.3 \\
\hline Age (Average) & 36.1 & 36.6 & $3.80 \%$ \\
\hline Asthma & $3.40 \%$ & $4.10 \%$ & $6.90 \%$ \\
\hline Diabetes & $7.40 \%$ & $10.60 \%$ & $10.90 \%$ \\
\hline Hypertension & $11.30 \%$ & $13.80 \%$ & $13.80 \%$ \\
\hline Obesity & $13.80 \%$ & $3.60 \%$ & $5.10 \%$ \\
\hline
\end{tabular}

Table 3: Percentage that left their house during the confinement.

\begin{tabular}{|c|c|c|}
\hline \multicolumn{1}{|c|}{ Week } & Left the House \\
\hline $26-M a r$ & $3-A p r$ & $17.91 \%$ \\
\hline $4-\mathrm{Apr}$ & $10-\mathrm{Apr}$ & $5.26 \%$ \\
\hline $11-\mathrm{Apr}$ & $17-\mathrm{Apr}$ & $4.16 \%$ \\
\hline $18-\mathrm{Apr}$ & $24-\mathrm{Apr}$ & $6.07 \%$ \\
\hline $25-\mathrm{Apr}$ & 1 -May & $3.80 \%$ \\
\hline 2-May & 8-May & $4.51 \%$ \\
\hline 9-May & 15-May & $3.76 \%$ \\
\hline $16-$ May & 22-May & $3.01 \%$ \\
\hline 23-May & 31-May & $4.30 \%$ \\
\hline
\end{tabular}


Table 4: Health measures followed by respondents during the pandemics.

\begin{tabular}{|c|c|}
\hline Health measures & Percentage \\
\hline Hand wash with water and soap & 96.1 \\
\hline Quarantine & 94.4 \\
\hline Use of face masks & 73.7 \\
\hline Change of shoes on returning to the house & 66.9 \\
\hline Change of clothes on returning to the house & 54.5 \\
\hline Use of masks or glasses & 11 \\
\hline Change of clothes and shoes on returning to the house & 10.7 \\
\hline Use of gloves & 9.9 \\
\hline None of the measures & 0.3 \\
\hline
\end{tabular}

Table 5: Reasons for leaving the home by the respondents

\begin{tabular}{|c|c|}
\hline & Percentage of those who left \\
\hline Work & $42.07 \%$ \\
\hline Purchase of basic supplies & $44.23 \%$ \\
\hline Work in the hospital & $27.37 \%$ \\
\hline Work in a doctor's office & $8.77 \%$ \\
\hline Use of bus as transport means & $59.50 \%^{*}$ \\
\hline Use of subway or underground as transport means & $32.86 \%^{*}$ \\
\hline Use of private vehicle (Taxi or Uber) & $29.97 \%^{*}$ \\
\hline
\end{tabular}

Note: *Those who used public transport

Table 6: Symptoms reported by the respondents during the pandemics.

\begin{tabular}{|c|c|c|c|}
\hline \multicolumn{2}{|c|}{ Week } & \# patients with Symptoms & \% patients with Symptoms \\
\hline $26-M a r$ & 3-Apr & 1162 & 19.2 \\
\hline $4-\mathrm{Apr}$ & $10-\mathrm{Apr}$ & 561 & 5 \\
\hline $11-\mathrm{Apr}$ & $17-\mathrm{Apr}$ & 642 & 4.7 \\
\hline $18-\mathrm{Apr}$ & $24-\mathrm{Apr}$ & 616 & 4.5 \\
\hline 25-Apr & 1-Apr & 409 & 4.2 \\
\hline 2-Apr & 8-Apr & 462 & 5.2 \\
\hline 9-Apr & 15-Apr & 301 & 4.3 \\
\hline 16-May & 22-May & 369 & 4.1 \\
\hline 23-May & 31-May & 277 & 5.9 \\
\hline
\end{tabular}

Table 7: Symptoms reported respondent population during the pandemics.

\begin{tabular}{|c|c|c|}
\hline Symptoms & No. & Percentage of Cases with Symptoms \\
\hline Cough & 1349 & 31.35 \\
\hline Headache & 1566 & 28.71 \\
\hline Odynophagia & 1235 & 28.24 \\
\hline Rhinorrhea & 1215 & 19.54 \\
\hline Asthenia & 841 & 6.58 \\
\hline Anosmia & 201 & \\
\hline
\end{tabular}


Table 8: Treatment used by the respondents during the pandemics.

\begin{tabular}{|c|c|c|}
\hline & No. & Percentage \\
\hline Acetaminophen & 897 & 0.98 \\
\hline Aspirin & 351 & 0.38 \\
\hline Vitamins & 290 & 0.32 \\
\hline Loratidine & 238 & 0.26 \\
\hline Ibuprofen & 185 & 0.2 \\
\hline Salbutamol & 144 & 0.16 \\
\hline
\end{tabular}

\section{Discussion}

In this work, we analyze some of the measures, for instance hand washing and confinement, proposed by Mexican Health Sector, in a specific group of the population (the Faculty of Medicine) who in the face of the absence of approved therapeutic strategies by any drug regulatory agency in the world or a vaccine to prevent the disease, have as the only effective measure physical isolation and reduction of population mobility Zhong et al. [9]. It was observed that of a total of 20,998 people that responded the survey, the highest participation was women (55.5\%). Of the entire population of respondents, $94.4 \%$ opted for confinement. Likewise, a high percent (96.1\%) carried out constant hand-wash. These measures that are within the recommendations of the WHO are performed by most of our population. Recently, the use of mouth mask by sick or non-sick people has turned out to be the main measure to avoid the spread of the virus and being infected NIOSH [10]. In our study, only $73.7 \%$ took the precaution of using it. The situations reported on the reasons that made the people to break the confinement were replenishment of basic home supply $(44.23 \%)$, work questions (42\%) and health works (27.37\%). In spite of the fact that the health authorities of many countries such as Mexico exempted companies with non-essential activities to work from home, a factor observed that impede strict fulfillment of the confinement is work. This fact goes hand in hand with the use of any kind of transport means to get to the work. We consider that for the containment of the disease, these two factors are crucial determinant. In countries like New Zealand, Singapore and Germany, the use of public transport was restricted at the beginning of confirmed cases Medrxiv [11]. In our study, of the activities that impede the people to keep the confinement, the most important was work. A total of $59.50 \%$ of these people used public transport. In Mexico, the beginning of changes in mobility in strategic points in public transport started in April Semovi (2020), a time when the accumulated average confirmed cases was 11,600 J Hopkins [5]. In this study, we observed that the fulfillment of the recommendations declared by the health authorities of each region or country does not guarantee the end of COVID-19 cases in spite of respecting confinement and keeping the hygienic measures. In our survey, $4.9 \%$ of the respondents had respiratory symptoms in the time of study. The most frequent symptoms were cough, headache and odynophagia, which corresponds to the clinical picture of SARSCoV-2 Matthew [12]. It is important to clarify that confirmatory test for SARS-CoV-2 was not performed in these cases. The drugs used to alleviate the symptoms are non-steroid anti-inflammatory drugs such as acetaminophen, acetylsalicylic acid and ibuprofen, vitamin supplements, loratidine and salbutamol. It should be noted that the measures of prevention and confinement are directed to all the population, nevertheless, the behavior of the disease indicates that there are group of people with chronic degenerative diseases susceptible to acquire the infection or to present a more severe picture Yang et al. [13].

In this survey, $10.9 \%$ of the people suffer arterial hypertension and $13 \%$ diabetes mellitus, both considered as a risk factor for SARS-CoV-2 infection. Although the percentage is not the majority in our study, it is important to know the situation and to emphasize the need for confinement to these people. At global level, tools have been designed to assess the behavior Wolf et al. $[14,15]$ knowledge, changes in lifestyle, prevention measures and risks of COVID-19 disease [13-19]. There is little evidence on the results of the prevention and confinement measures in the population. A way in which such results can be reflected is in the number of contagions as observed in different countries. Although the outbreak originated in China, the government and the health ministry this country worked together to moderate the number of infections and deaths associated to SARS.CoV-2. From January 11 to June 26, confirmed cases and deaths registered were 85,000 and 4,648 respectively in this country WHO (2020). The measures they took were more drastic and included international and national border closure as well as entry and exit into and from Wuhan province, the epicenter of the outbreak; isolation of cases; closure of schools, airlines; and actions that are impossible to accomplish in other countries Kupferschmidt et al. [20]. Most of the Chinese residents have knowledge on COVID-19 and optimistic attitudes towards prevention and self-care Zhong [9].

In contrast, countries like Brazil with a daily average confirmed cases of 2,138 and which, despite having a better proportion of test of 14/100,000 inhabitants, have not had a good result in deflecting the number of contagions J Hopkins [21]. A limiting factor in the analysis of the situation is the few number of technical reports 
made known to the general population and the adjustment of the measures taken depending on the number of confirmed cases. A similar case is the United State of America with daily average confirmed cases of 16,811 J Hopkins [5], and where the measures of confinement were adapted according to government and health guidelines. On the beginning of May, places such as beaches, gyms and restaurants were reopened by the government. Two weeks after, there was a rebound in the total of confirmed cases that reached about 1,000,000 J Hopkins [5]. Another important comparison with the same USA is based on survey with 630 adults between 23 and 88 years who were living with one or more chronic ailments. In this survey, $21.9 \%$ informed that the pandemics had little impact or effect on their daily life Wolf [13]. FacMed data revealed that among the respondents, more than $10 \%$ had chronic diseases such as hypertension and obesity. This figure is less than the national estimates, since it includes people under 25 years old, unlike the survey in USA; and as previously mentioned in our case, the measures taken by our population was hand-wash, confinement and use of mouth mask. In Mexico, the first two confirmed cases of infection by SARS-CoV-2 was registered on February 28, 2020 Health Ministry [22] and the first measure taken was the isolation of these first imported cases. It was not until mid-March that the health authorities called for confinement, closure of schools, theaters, movie theaters, bars, gyms and other non-essential activities. These measures were maintained until June 1 when partial return to the new-lifestyle-reality was declared. Two weeks after, a daily average confirmed case of 1,091 was registered J Hopkins [5].

\section{Conclusion}

The emergency of SARS-CoV-2 [23] in different countries and its serious effect on health of the population led to physical-distance decision-making as one of the most effective strategies to avoid the transmission of the virus. To assess the scope of the measures implemented, it was necessary the use of any standard assessment tool and the most easy-to-perform adequate tool is a survey as the one analyzed in this work. The FacMed survey shows the discipline of her community and her interest for collective health [24-25]. The dissemination of useful information to prevent infections such as the use of a mouth and face mask, physical distancing and direct medical orientation phone lines and others should continue to be re-enforced.

\section{Declaration}

\section{Competing Interests}

The authors declare that there are no competing interests.

\section{Funding}

This research did not receive any specific grant from funding agencies in the public, commercial, or not-for-profit sectors.

\section{References}

1. Troeger C (2015) Estimates of the global, regional, and national morbidity, mortality, and aetiologies of lower respiratory tract infections in 195 countries: a systematic analysis for the Global Burden of Disease Study 2015. Lancet Infectious Disease 17(11): 1133-1161.

2. World Health Organization (a) [Organización Mundial de la Salud.

3. World Health Organization (b) Organización mundial de la salud.

4. Echevarria Castro N, AR Fernandez Aristi (2020) About a lockdown: are the novel etiological therapies for the sars-cov-2 effective? Annal Case Report Review 3: 1-6.

5. Johns Hopkins (a) Coronavirus resource center.

6. (2020) Mitigation or containment, the two options for governments to address the coronavirus.

7. Koo JR, Alex R Cook, Minah Park, Yinxiaohe Sun, Haoyang Sun, et al. (2020) Interventions to mitigate early spread of SARS-CoV-2 in Singapore: a modelling study. Lancet Infectious Disease 20(6): 678-688.

8. Reza SA, M Marcello, C Ndaona (2020) COVID-19 Epidemic in Switzerland: Growth Prediction and Containment Strategy Using Artificial Intelligence and Big Data. MEDRXIV.

9. Zhong (2020) Knowledge, attitudes, and practices towards covid-19 among chinese residents during the rapid rise period of the covid-19 outbreak: a quick online cross-sectional survey. International Journal of Biologic Science 16(10): 1745-52.

10. (2020) National Institute for Occupational Safety and Health (NIOSH). Use of respirators and surgical masks for protection against healthcare hazards.

11. Medrxiv (2020) The goal of these strategies is to slow the spread of infection and reduce the intensity of the epidemic ("flatten the curve") (3), thus reducing risk of overwhelming health systems and buying time to develop treatments and vaccines.

12. Matthew JC (2020) Epidemiology, clinical course, and outcomes of critically ill adults with COVID-19 in New York City: a prospective cohort study. The Lancet 395: 1763-1770.

13. Yang XY Yu, J Xu (2020) Clinical course and outcomes of critically ill patients with SARS-CoV-2 pneumonia in Wuhan, China: a single-centered, retrospective, observational study. Lancet Respiratory Medicine 8(5): 475-481.

14. Wolf MS (2020) Awareness, attitudes, and actions related to covid-19 among adults with chronic conditions at the onset of the u.s. outbreak: a cross-sectional survey. Annals of Internal Medicine 20: 1239.

15. Bodas M, K Peleg (2020) Self-isolation compliance in the covid-19 era influenced by compensation: findings from a recent survey in Israel. Health Affairs (Millwood) 39(6).

16. Geldsetzer P (2020) Using of rapid online surveys to assess perceptions during infectious disease outbreaks: a cross-sectional survey on Covid-19. Journal of Medical Internet Research 22: 1-13.

17. Taghrir MH, R Borazjani, R Shiraly (2020) COVID-19 and Iranian medical students; a survey on their related-knowledge, preventive behaviors and risk perception. Archives Iranian Medicine 23(4): 249-54.

18. Khan S, Mahjabeen Khan, Khizra Maqsood, Tanveer Hussain, Noor-ulHuda, et al. (2020) Is Pakistan prepared for the COVID-19 epidemic? A questionnaire based survey. Journal of Medical Virology.

19. Moro M, Giacomo Pietro Vigezzi, Michele Capraro, Anna Biancardi, Paola Nizzero et al. (2020) 2019-novel coronavirus survey : knowledge and attitudes of hospital staff of a large Italian teaching hospital. Acta Biomedica 91(11): 29-34. 
20. Kupferschmidt K, J Cohen (2020) Can China's COVID-19 strategy work elsewhere? Science 367(6482): 1061-1062.

21. Johns Hopkins (b), Mexico 2/100,000

22. (2020) Health Ministry. Coronavirus technical releases Health Secretary. Coronavirus technical releases.

23. (2020) Characteristics of and important lessons from the coronavirus disease 2019 (COVID-19). Outbreak in china: summary of a report of 72314 cases from the chinese center for disease control and prevention. Journal of American Medical Association 323(13): 1239-1242.
24. (2020) Infection prevention and control during health care when novel coronavirus (nCoV) infection is suspected. Interim Guidance. Geneva: World Health Organization.

25. (2020) Standard precautions in health care. Geneva: World Health Organization. 